Diese Pauschale ist unabhängig von der Zahl der Vertragsärzte in der Praxis und wird $a b$ dem 3. Quartal 2018 reduziert.

Wenn die Praxen ein mobiles Kartenterminal finanziert bekommen wollen, müssen sie Voraussetzungen erfüllen. Im Quartal des Antrags oder im Vorquartal müssen mindestens drei Besuche nach den EBM-Nrn. 01410 01 413, 01 415, 01418 oder 05230 abgerechnet werden. Auch Ärzte, die ein Heim betreuen, haben Anspruch darauf - sie müssen an einem Kooperationsvertrag gemäß $\S 119 b$ SGB V teilnehmen, der den Vorgaben der Anlage 27 zum BundesmantelvertragÄrzte (BMV-Ä) entspricht. Ebenfalls erstattet werden die Kosten der SMC-B-Karten, die für den Konnektor und jedes mobile Kartenterminal benötigt werden, sowie die Hälfte der monatlichen Kosten für den elektronischen Heilberufeausweis (HBA-Smartcard).

\section{Tab. 1 Erstattung von Kosten für Vertragsarztpraxen im Rahmen der Einführung der Telematikinfrastruktur}

\begin{tabular}{|c|c|c|c|c|c|}
\hline \multicolumn{2}{|l|}{ Pauschale } & \multicolumn{3}{|c|}{ Betrag in Euro } & \multirow{2}{*}{$\begin{array}{l}\text { Zahlungs- } \\
\text { weise }\end{array}$} \\
\hline & Quartal & 1-3 Ärzte & 4-6 Ärzte & > 6 Ärzte & \\
\hline \multirow{5}{*}{ Erstausstattung } & $3 / 2017$ & $3.055 €$ & $3.490 €$ & $3.925 €$ & \multirow{5}{*}{ einmalig } \\
\hline & $4 / 2017$ & $2.793 €$ & $3.228 €$ & $3.663 €$ & \\
\hline & $1 / 2018$ & $2.557 €$ & $2.992 €$ & $3.427 €$ & \\
\hline & $2 / 2018$ & $2.345 €$ & $2.780 €$ & $3.215 €$ & \\
\hline & $a b 3 / 2018$ & $1.155 €$ & $1.590 €$ & $2.025 €$ & \\
\hline \multirow{2}{*}{ Betriebskosten } & bis $2 / 2018$ & & $298 €$ & & \multirow{2}{*}{ je Quartal } \\
\hline & $a b 3 / 2018$ & & $248 €$ & & \\
\hline \multicolumn{2}{|c|}{ mobiles Kartenterminal } & & $350 €$ & & einmalig \\
\hline \multicolumn{2}{|c|}{ Aufwendungen zum Systemstart } & & $900 €$ & & einmalig \\
\hline \multicolumn{2}{|l|}{ SMC-B-Smartcard } & & $23,25 €$ & & je Quartal \\
\hline \multicolumn{2}{|l|}{ HBA-Smartcard } & & $11,63 €$ & & je Quartal \\
\hline
\end{tabular}

\title{
Häusliches Arbeitszimmer von der Steuer absetzen
}

Nach einem Urteil des Bundesfinanzhofs (BFH) können niedergelassene Ärzte ein häusliches Arbeitszimmer steuermindernd geltend machen, wenn sich in ihrer Praxis kein geeigneter Büroraum einrichten lässt (Az.: III R 9/16). Im konkreten Fall hatte ein selbstständiger Logopäde geklagt, der in angemieteten Räumen an unterschiedlichen Orten praktizierte. Diese lagen allerdings bis zu $47 \mathrm{~km}$ von seiner Wohnung entfernt und wurden überwiegend von seinen vier Angestellten genutzt.

Verwaltungsarbeiten erledigte der Mann daher in seinem häuslichen Arbeitszimmer. Die anteiligen Miet- und Nebenkosten setzte er von der Steuer ab - was das Finanzamt ihm verweigerte. Der Streit ging vor Gericht, zog sich durch alle Instanzen und endete nun zugunsten des Logopäden.

\section{MMW-KOMMENTAR}

Der Fall kann in geeigneten Fällen auf Ärzte übertragen werden. Laut BFH ist es dem Kläger nicht zuzumuten, seine Büroarbeiten in der Praxis zu erledigen. Die Nutzung des Arbeitsplatzes als Büro sei hier so beschränkt, dass er gar keine andere Wahl habe, als zu Hause zu arbeiten. Er durfte deshalb die Aufwendungen für das häusliche Arbeitszimmer bis zum Höchstbetrag von 1.250 Euro im Jahr von der Steuer abziehen.

Der BFH machte allerdings klar, dass stets der Einzelfall zu betrachten sei - vor allem die Praxisorganisation. So ist etwa die Wahrung der Vertraulichkeit bei der Abrechnung in einem offenen Praxiskonzept wie dem des Logopäden schwierig. Die Verlagerung solcher Aufgaben auf den Feierabend, wie es das Finanzamt vorgeschlagen hatte, hielten die BFH-Richter für nicht zumutbar.

Ein weiteres Urteil des Finanzgerichts Mün-

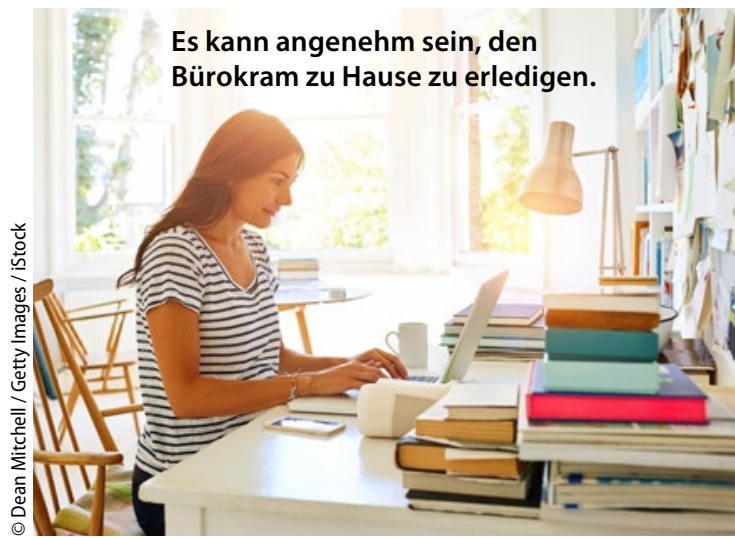

chen festigt diesen Anspruch, wenn z. B. ein Arbeitszimmer für Bereitschaftsdienste am Wochenende benötigt wird. Im konkreten Fall ging es um einen Projektleiter für internationale Bauprojekte, der auch an Wochenenden erreichbar sein musste. Das Büro bei seinem Arbeitgeber konnte er dafür nicht nutzen, weshalb er zu Hause arbeitete. Das Finanzgericht hat den Anspruch anerkannt (Az.: 15 K 439/15). Der Fall ist auch auf Ärzte übertragbar, die zu Hause einen eigenen Arbeitsraum für Bereitschaftsdienste z. B. an den Wochenenden nutzen. 